\title{
Résistances aux herbicides chez les mauvaises herbes
}

\author{
H Darmency, J Gasquez
}

INRA, Laboratoire de Malherbologie, BV 1540, 21034 Dijon Cedex, France

(Reçu le 8 mars 1990; accepté le 27 mai 1990)

\begin{abstract}
Résumé - L'usage inconsidéré des herbicides pour un désherbage systématique des grandes cultures a conduit à l'apparition de mauvaises herbes résistantes. Huit familles d'herbicides sont concernées par ce problème. Plus de 60 espèces montrent des populations résistantes qui sont réparties dans tous les pays industrialisés. Monoculture et répétition des mêmes traitements sont à l'origine de ce phénomène. Dans certains cas, la sélection pour la résistance à un herbicide a aussi conduit à la résistance pour d'autres herbicides de modes d'action parfois différents. Des exemples sont décrits faisant le point de nos connaissances et en essayant de dégager les stratégies propres à combattre ces résistances.
\end{abstract}

mauvaise herbe / herbicide / résistance

Summary - Herbicide resistances in weeds. The indiscriminate use of herbicides for selective weed control in major crops has led to the appearance of resistant weed biotypes. There are now 67 species that have evolved biotypes resistant to one or more herbicides. This includes 19 grasses and 48 broadleaf weed species. Nine herbicide families are involved in this connection. Herbicide resistance is now found in virtually all industrial countries. It is estimated that the land infested with triazine resistant weeds exceeds 2 million ha mostly in the USA, Canada and Europe. Repetition of the same crop and the same herbicide on a field is largely responsible for this phenomenon. In a few cases, selection for resistance to one herbicide has also led to resistance to other herbicides sometimes having different modes of action (cross-resistance). Control of triazine resistant weeds has been satisfactorily achieved, however increasing the cost of the weed management strategy. It is doubtful whether new resistances, especially in the case of cross resistances, can be managed as successfully as triazine resistance was in the past. As triazine resistance is the first and the most documented case of herbicide resistance, one third of this paper is devoted to its history, distribution (table I), mechanism, inheritance, physiology and fitness consequences. Other examples of herbicide resistance are described (table II) as well as discussion of strategies to combat resistance.

weed / herbicide / resistance

\section{INTRODUCTION}

Les moyens mécaniques, qui très longtemps ont assuré un désherbage non sélectif des mauvaises herbes annuelles, ont été remplacés depuis près de 40 ans par des herbicides de plus en plus sélectifs. Ces herbicides sont devenus indispensables à notre agriculture moderne. Ils représentent $63 \%$ du total des ventes de pesticides destinés à l'agriculture aux États-Unis, soit 17 milliards de francs en 1986 (LeBaron et McFarland, 1990) et seulement $45 \%$, soit 4 milliards de francs en 1987, en France (My et Lorelle, 1988).

Le but recherché lorsqu'on utilise des herbicides en agriculture est généralement de détruire les mauvaises herbes sans affecter la plante cultivée, pour qu'elle profite au mieux de l'élimination des adventices. Cette action qui va altérer un ou plusieurs mécanismes physiologiques, doit nécessairement être sélective et s'exercer d'une manière différentielle entre culture et mauvaises herbes. Ceci implique que différentes espèces ne vont pas réagir de la même façon, y compris à l'intérieur d'une même famille botanique, par exemple des graminées dans les céréales, des Brassicacées dans le colza. On peut aussi penser que la variabilité infraspécifique favorise des réponses différentes entre les individus d'une même espèce de mauvaise herbe.

Aujourd'hui, il existe une importante littérature décrivant le comportement particulier de certains 
biotypes de mauvaises herbes après traitement aux herbicides (LeBaron et Gressel, 1982). Ce comportement peut varier depuis de simples écarts de réponse aux doses normales utilisées, dus à des différences de phénologie ou de vigueur, jusqu'à des résistances très élevées même à 100 fois la dose conseillée, dues à des systèmes de détoxication très efficaces ou à des mutations des cibles. Plus précisément, nous entendons par plante résistante une plante qui survit à une concentration d'herbicide qui détruit complètement des plantes provenant de populations témoins jamais soumises à la pression de sélection de l'herbicicide. Cette définition ne s'applique donc pas aux espèces pour lesquelles les herbicides n'ont jamais eu d'effets (insensibilité innée). Elle exclut les artefacts liés à des traitements défectueux et implique la notion d'un témoin de référence et la transmission au moins partielle dans la descendance, ce qui traduit implicitement la réalisation d'une expérience en vue de rechercher un mécanisme de résistance. Certains auteurs préfèrent le concept de tolérance à celui de résistance pour caractériser des plantes qui, aux doses normalement utilisées, présentent des symptômes passagers, ce qui présagerait d'une résistance faible (Lutman et Lovegrove, 1985; Andersen et Gronwald, 1987; Stanger et Appleby, 1989). Nous n'utiliserons jamais ce terme car il est source de malentendu. En effet, de tels symptômes peuvent également être l'expression de résistances très élevées mais dont l'action peut être différée. II nous apparaît enfin nécessaire de mettre en garde contre la définition de populations résistantes caractérisées par une mortalité moyenne, car elles peuvent être constituées d'un mélange de plantes résistantes et sensibles. L'évolution de la réponse de la population après plusieurs traitements dépendra de la proportion de plantes résistantes et sensibles (éventuellement plusieurs types et niveaux de résistants), du type de résistance et de son hérédité (mono ou polygénique, nucléaire ou cytoplasmique) et du mode de reproduction (auto ou allogame) de l'espèce. Il est donc clair que les cas de résistance doivent être décrits dans des conditions standardisées à l'aide de plantes résistantes bien identifiées, en précisant leur fréquence dans les populations.

Nous nous proposons de passer en revue les différents cas de mauvaises herbes résistantes aux herbicides apparus jusqu'à ce jour, en développant plus en détail le cas de la résistance aux triazines, le plus ancien et le mieux étudié. Par ailleurs, seuls les cas ayant entraîné des infesta- tions gênantes pour l'agriculteur seront retenus. On peut trouver dans la littérature d'autres données sur des types de plantes plus résistantes à certains produits, mais il s'agit de résultats d'études sur la variabilité génétique intraspécifique et non de cas concrets d'infestations non maîtrisées. Dans plusieurs cas, l'origine des populations les moins sensibles correspond aux zones où le désherbage était utilisé : Cirsium arvense (Folgelfors, 1979) et Tripleurospermum inodorum (Ellis et Kay, 1975) pour le MCPA, Avena fatua pour le triallate (Jana et Naylor, 1982), Poa annua pour l'aminotriazole (Bulke et al, 1984). Ceci laisse penser à une sélection de ces populations pour une meilleure survie à l'herbicide sans toutefois mettre en œuvre un mécanisme tel que ces plantes résistent à 100\% aux doses agricoles d'utilisation. D'autres cas de différences intraspécifiques pour la réponse aux herbicides ont été signalés (Le Baron et Gressel, 1982). II s'agit essentiellement de différences mises en évidence à la suite d'expériences réalisées afin d'interprêter les variations des résultats au désherbage. Ainsi, des chercheurs se sont demandés s'il n'existait pas une composante génétique qui entraînait une hétérogénéité du matériel végétal, par exemple, d'Avena fatua vis-àvis du barbane (Price et al (1983) ou Convolvulus arvensis vis-à-vis du glyphosate (de Gennaro et Weller, 1984).

\section{LES TRIAZINES}

\section{Historique}

La majorité des herbicides de la famille des triazines symétriques est utilisée en pré-levée et donc absorbée par les racines alors que leur cible unique se trouve dans les chloroplastes. Les plantes peuvent échapper à l'herbicide parce que les racines n'absorbent pas dans la zone du sol où se trouve l'herbicide. Mais en réalité la sélectivité de ces produits est fondée sur l'aptitude de certaines espèces comme le maïs à détoxifier les produits avant qu'ils n'atteignent les chloroplastes. Ainsi, pour les chlorotriazines, il existe l'hydroxylation, la N-déalkylation et surtout la conjugaison avec le glutathion qui est la voie la plus importante chez le maïs (Shimabukuro et al, 1966).

La plupart des dicotylédones ne détoxifient pas ou très peu. Chez les graminées, seules les Panicoïdées et quelques Éragrostidées détoxi- 
fient dans des proportions notables. Mais il existe de très grandes variabilités entre les genres, entre les espèces et même à l'intérieur d'une même espèce (Warwick, 1976; Karim et Bradshaw, 1968). Des populations d'Echinochloa crusgalli issues de maïs traités plusieurs années étaient globalement plus résistantes que des populations jamais traitées (Gasquez et Compoint, 1973), mais cela n'a pas conduit à la constitution de populations d'individus résistant à des doses très élevées. En revanche, une dicotylédone est devenue résistante par détoxication, Abutilon theophrasti (Andersen et Gronwald, 1987). Dans ce cas, il s'agit d'une augmentation de l'activité de 2 à 4 fois de la glutathion $S$-transférase qui est héritée via un simple gène nucléaire. Mais ce mécanisme semble encore assez isolé. Mis à part l'observation de biotypes résistants de Lolium rigidum sur des voies ferrées en Australie (Powles et Howat, 1990) et d'Amaranthus lividus dans des maïs en Suisse (Beuret, 1988), dont les mécanismes restent encore inexpliqués, c'est un autre type de résistance aux triazines qui s'est répandu chez les mauvaises herbes : la résistance chloroplastique.

En 1968, après 10 ans d'utilisation des triazines 1 ou 2 fois par an, Ryan découvrait dans des pépinières de l'état de Washington une population de Senecio vulgaris résistant à de très fortes doses de simazine (Ryan, 1970). En 1974, Peabody signalait encore aux États-Unis, une population d'Amaranthus sp résistant à de fortes doses d'atrazine. Puis d'autres populations résistantes ont été observées dans de nombreux pays : dès 1978 nous signalions en France une population résistante de Chenopodium album et de Poa annua (Ducruet et Gasquez, 1978). C'est seulement en 1977 que l'on a montré que des chloroplastes isolés des plantes résistantes n'étaient pas affectés par l'atrazine et supportaient des doses 1000 fois supérieures à celles qui bloquent les sensibles (Souza-Machado et al, 1977).

\section{Distribution}

En 1981, nous avions déjà signalé en France des populations résistantes chez 20 espèces (Gasquez, 1981); heureusement la liste ne s'est pas allongée depuis : 4 n'ont été observées qu'en France (Gasquez et Darmency, 1990). Les dernières observations font état d'environ 50 espèces dans le monde entier, toutes annuelles ou se comportant comme telles (Le Baron et Mc
Farland, 1990). Celles-ci se répartissent en plus de 30 espèces dicotylédones et une quinzaine de graminées, dont quelques unes peuvent aussi détoxifier les triazines.

Ces populations se rencontrent aux ÉtatsUnis (17 états), au Canada et dans la majorité des pays européens, ainsi qu'en Israël (tableau I). La culture la plus touchée est le maïs, mais on trouve des résistants dans le vignoble, les pépinières, les vergers, les oliviers, etc. On les rencontre aussi dans des zones non cultivées : des zones industrielles, voies ferrées, routes, rues de villes. En fait, la résistance s'est développée dans tous les milieux où l'on utilise régulièrement les triazines.

Par exemple, en France, en 1981, une enquête nous avait montré qu'environ 250000 ha de maïs étaient envahis par au moins une espèce résistante (Gasquez et al, 1982). Aujourd'hui, il est plus difficile de mesurer cette extension à cause des traitements systématiques de post-levée dirigés spécialement contre ces plantes (environ 1,4 million d'ha en 1989). On peut l'estimer à au moins 500000 ha de maïs (sur un total de 3 millions) distribués dans la quasi-totalité des régions productrices. Plusieurs milliers d'hectares de vigne sont aussi touchés dans tous les grands vignobles.

En France, mais aussi dans le monde, l'espèce présentant le plus de populations résistantes est Chenopodium album. Cependant, la distribution varie beaucoup d'une espèce à l'autre. Ainsi, Solanum nigrum, les amaranthes et Senecio vulgaris présentent aussi de nombreuses populations résistantes. En revanche, la résistance chez Polygonum lapathifolium, $P$ persicaria et Sonchus asper est limitée à quelques populations, voire une seule (Le Baron et McFarland, 1990). Pour certaines espèces, les foyers de résistance sont en régression du fait de la lutte dirigée contre ces mauvaises herbes comme Chenopodium polyspermum en France, ou même, ont disparu comme pour Stellaria media en RFA.

\section{Mécanisme}

Depuis longtemps, il est connu que les triazines bloquent la photosynthèse au niveau du transfert d'électrons du photosystème II. Cependant, c'est grâce à l'étude des mutants résistants que l'on sait qu'elles entrent en compétition avec une quinone pour s'attacher à une protéine membranaire : la protéine $32 \mathrm{kDa}$. 
Tableau I. Liste des espèces montrant des populations résistantes aux triazines après mutation chloroplastique, année de la première découverte et pays infestés. A : Autriche; AU : Australie; B : Belgique; $\mathrm{BG}$ : Bulgarie; $\mathrm{CH}$ : Suisse; CND : Canada; CS : Tchécoslovaquie; D : RFA; DDR : RDA; DK : Danemark; E : Espagne; ET : Egypte; F : France; GB : Royaume-Uni; H : Hongrie; I : Italie; IL : Israël; J : Japon; NL : Pays-Bas; NZ : Nouvelle-Zélande; PL : Pologne; R : Roumanie; USA : États-Unis; YU : Yougoslavie.

\begin{tabular}{|c|c|c|}
\hline Espèce & Année & Pays \\
\hline \multicolumn{3}{|l|}{ Dicotylédones } \\
\hline Amaranthus albus & 1988 & $E$ \\
\hline Amaranthus blitoides & 1983 & E, IL \\
\hline Amaranthus bouchonii & 1981 & $\mathrm{CH}, \mathrm{F}, \mathrm{I}, \mathrm{H}$ \\
\hline Amaranthus cruentus & 1978 & $\mathrm{I}, \mathrm{E}$ \\
\hline Amaranthus hybridus & 1972 & $\begin{array}{c}\mathrm{CH}, \mathrm{E}, \mathrm{F}, \mathrm{H}, \\
\mathrm{I}, \mathrm{IL}, \mathrm{USA}\end{array}$ \\
\hline Amaranthus lividus & 1978 & $\mathrm{CH}, \mathrm{E}, \mathrm{F}$ \\
\hline Amaranthus powellii & 1968 & CND, USA \\
\hline Amaranthus retroflexus & 1973 & $\begin{array}{c}A, B, C N D, C H, C S, \\
F, H, R, \text { USA, YU }\end{array}$ \\
\hline Ambrosia artemisiifolia & 1975 & CND, USA, YU \\
\hline Arenaria serpylifolia & 1981 & $\mathrm{~F}$ \\
\hline Bidens tripartita & 1976 & A \\
\hline Brassica campestris & 1977 & CND \\
\hline Chamomilla suaveolens & 1988 & $\mathrm{~GB}$ \\
\hline Chenopodium album & 1970 & $\begin{array}{c}\text { A,B, BG, CND, CH, } \\
\text { CS, D, DDR, E, F, } \\
\text { H, I, NL, PL, YU, GB }\end{array}$ \\
\hline Chenopodium ficifolium & 1978 & $\mathrm{CH}, \mathrm{D}, \mathrm{NL}$ \\
\hline Chenopodium missouriense & 1978 & USA \\
\hline Chenopodium polyspermum & 1979 & $\mathrm{CH}, \mathrm{D}, \mathrm{F}, \mathrm{H}, \mathrm{R}$ \\
\hline Chenopodium strictum & 1978 & CND \\
\hline Conyza bonariensis & 1987 & $E$ \\
\hline Conyza canadensis & 1978 & $\begin{array}{l}\mathrm{CH}, \mathrm{CS}, \mathrm{DK}, \mathrm{F} \\
\mathrm{H}, \mathrm{PL} \mathrm{GB}, \mathrm{R}, \mathrm{YU}\end{array}$ \\
\hline Epilobium ciliatum & 1982 & $\mathrm{~B}, \mathrm{DK}, \mathrm{NL}, \mathrm{GB}$ \\
\hline Epilobium tetragonum & 1981 & $D, F$ \\
\hline Fallopia convolvulus & 1977 & $A, D, N L, U S A$ \\
\hline Galinsoga ciliata & 1980 & NL, D \\
\hline Kochia scoparia & 1976 & USA \\
\hline Myosoton aquaticum & 1983 & $\mathrm{D}$ \\
\hline
\end{tabular}

La structure primaire de cette molécule, reconnue par un marquage de photo-affinité (Pfister et al, 1981) a été inférée à partir de la séquence de son gène $(p s b A)$, localisé sur l'ADN chloroplastique (Rao et al, 1983). Par des simulations, un modèle de la structure tertiaire de cette molécule a été établi : elle traverse la membrane du thylakoïde par 5 hélices et présente à l'extérieur une grande boucle partiellement en hélice qui est le site de fixation des herbicides (Trebst, 1987).

Chez les plantes résistantes, le séquençage du gène $p s b A$ a révélé que seule une mutation de la première base du triplet codant pour l'acide aminé en position 264 est exprimée, ce qui transforme un résidu sérine en glycine (Hirshberg et Mc Intosh, 1983). Par la suite, ce gène a aussi été séquencé chez plusieurs autres plantes supérieures et dans chaque cas cette mutation a été systématiquement observée (Dar-

\begin{tabular}{|c|c|c|}
\hline Espèce & Année & Pays \\
\hline $\begin{array}{l}\text { Polygonum aviculare } \\
\text { Polygonum lapathifolium } \\
\text { Polygonum pensylvanicum } \\
\text { Polygonum persicaria } \\
\text { Senecio vulgaris }\end{array}$ & $\begin{array}{l}1987 \\
1978 \\
1988 \\
1979 \\
1968\end{array}$ & $\begin{array}{c}\text { NL, PL } \\
\text { CS, D, F, NL } \\
\text { USA } \\
\text { F, NL } \\
\text { B, BG, CND, D, } \\
\text { CH, CS, DK, F, } \\
\text { NL, GB, USA }\end{array}$ \\
\hline $\begin{array}{l}\text { Sinapis arvensis } \\
\text { Solanum nigrum }\end{array}$ & $\begin{array}{l}1983 \\
1978\end{array}$ & $\begin{array}{c}\text { CND } \\
\text { A, B, CH, D, E, F, I, } \\
\text { NL, R, GB, USA }\end{array}$ \\
\hline $\begin{array}{l}\text { Sonchus asper } \\
\text { Stellaria media } \\
\text { Viola arvensis }\end{array}$ & $\begin{array}{l}1981 \\
1974 \\
1988\end{array}$ & $\begin{array}{c}F \\
\mathrm{D} \\
\mathrm{GB}\end{array}$ \\
\hline \multicolumn{3}{|l|}{ Graminées } \\
\hline $\begin{array}{l}\text { Alopecurus myosuroïdes } \\
\text { Brachypodium distachyon } \\
\text { Bromus tectorum } \\
\text { Digitaria sanguinalis } \\
\text { Echinochloa crus galli } \\
\text { Lolium rigidum } \\
\text { Panicum capillare } \\
\text { Phalaris paradoxa } \\
\text { Poa annua } \\
\\
\text { Polypogon monspeliensis } \\
\text { Setaria faberi } \\
\text { Setaria glauca } \\
\text { Setaria viridis } \\
\text { Setaria v var major }\end{array}$ & $\begin{array}{l}1982 \\
1976 \\
1977 \\
1983 \\
1978 \\
1982 \\
1975 \\
1982 \\
1977\end{array}$ & $\begin{array}{c}\text { IL } \\
\text { IL } \\
F, \text { USA } \\
F, P L \\
A, F, P L, \text { USA } \\
\text { IL } \\
\text { USA } \\
\text { IL } \\
\text { A, B, CH, CS, D, } \\
\text { F, NL, GB, USA, } \\
\text { IL } \\
\text { USA } \\
\text { CND, E, F, USA } \\
F \\
F\end{array}$ \\
\hline
\end{tabular}

mency et Gasquez, 1990). La perte de la fonction alcool de la sérine en position 264 sur une partie libre de la protéine, entraîne une modification des potentiels d'oxydoréduction (Bowes et al, 1980; Demeter et al, 1985). Cela conduirait à la perte d'affinité de l'herbicide (Jursinic et Stemler, 1983). Enfin, le rôle de ce gène dans la résistance a été confirmé après avoir été introduit par génie génétique dans le génome nucléaire d'un Nicotiana chez lequel un effet de résistance à l'atrazine a été observé (Cheung et al, 1988).

La perte d'affinité de l'herbicide qui découle de la mutation est clairement mise en évidence par l'étude des doses d'atrazine qui inhibent $50 \%$ de l'activité des chloroplastes isolés in vitro : l'écart entre les sensibles et les résistants est très grand, et le rapport de l'activité des résistants à celle des sensibles (R/S) varie de 300 à 1200 selon les espèces (Gasquez, 1981). Les variations de réponse des chloroplastes peuvent 
aussi être observées à l'intérieur d'une espèce, comme Poa annua (Gasquez et Darmency, 1983). Au champ, les plantes résistantes ne semblent pas être affectées par $20 \mathrm{~kg} / \mathrm{ha}$ d'atrazine tandis que la dose létale est de 0,1 à $0,2 \mathrm{~kg} / \mathrm{ha}$ pour les sensibles, mais des différences peuvent exister entre espèces et même au sein d'espèces, comme chez Solanum nigrum (Zanin et al, 1981).

Le mécanisme de résistance est également efficace contre les autres chloro, méthoxy et méthylthio-S-triazines pour lesquelles le rapport R/S est compris de 50 à 500 (Souza-Machado et al, 1978a) : on parle alors de multi-résistance. On remarquera cependant que si le rapport $R / S$ est environ 50 pour la métribuzine (triazine asymétrique), les plantes résistantes ne sont pas pour autant très résistantes au traitement à dose normale : il peut exister un décalage important entre le test in vitro du site d'action et le comportement de la plante entière. De plus, les plantes résistent à la pyrazone mais pas à la bentazone. On observe aussi une certaine résistance à plusieurs urées substituées (monolinuron, linuron, fénuron), mais avec des rapports $R / S$ plus faibles que précédemment, de l'ordre de 3 à 15 (Ducruet et de Prado, 1982). La réponse à d'autres herbicides est très variée mais la nature de l'espèce influe peu sur la nature de la résistance croisée. La comparaison des chloroplastes peut laisser présager d'une résistance accrue des plantes résistantes aux uraciles et pyridazinones (Fuerst et al, 1986).

Enfin, notons qu'une certaine résistance au diclofop-méthyl a été observée chez les plantes résistantes aux triazines chez 2 graminées (Rubin et al, 1985). En revanche, une efficacité plus grande des nitrophénols (dinosèbe et DNOC) et de la bentazone a été généralement observée chez les biotypes résistants (Fuerst et al, 1986).

\section{Hérédité}

Très rapidement, il a été montré que ce caractère a une hérédité cytoplasmique : seule la plante mère peut transmettre la résistance (SouzaMachado et al, 1978b). Ceci s'est trouvé confirmé lorsque le gène psbA a été reconnu dans I'ADN chloroplastique.

Cependant, chez Solanum nigrum (Gasquez et al, 1981a), Poa annua (Darmency et Gasquez, 1981) et Chenopodium album (Gasquez et al, 1984), nous avons pu montrer qu'il y avait régu- lièrement une faible hérédité paternelle due au transfert exceptionnel d'organites cytoplasmiques à partir du pollen. Comme conséquence de cette transmission maternelle, on observe un effet de fondation de la population résistante avec un seul cytoplasme souvent lié à très peu de variabilité nucléaire. En effet, dans les champs, le polymorphisme des populations et le taux d'allogamie sont faibles, ce qui, lié à la sélection par l'herbicide conduit à des populations résistantes monomorphes (Gasquez et Compoint, 1981; Warwick et Marriage, 1982). Cette structure monomorphe se conservera d'autant mieux que la population est isolée, mais un nouveau polymorphisme pourra apparaître par échange génique dans les milieux où plantes sensibles et résistantes peuvent voisiner (Darmency et Gasquez, 1983).

\section{Reconnaissance des plantes résistantes}

Empiriquement, les populations résistantes se reconnaissent sur le terrain par le fait qu'elles résistent à n'importe quelle dose d'atrazine. Par exemple, $40 \mathrm{~kg} / \mathrm{ha}$ d'atrazine n'arrêtent pas le développement de chénopodes résistants produisant des taches d'une seule espèce dans un champ bien propre par ailleurs. Par comparaison, les plantes sensibles sont toutes détruites avec $200 \mathrm{~g} / \mathrm{ha}$. Au laboratoire, il existe plusieurs techniques de reconnaissance directement liées au mécanisme et à l'hérédité de la résistance :

- les résistants ayant un transfert d'électrons plus lent au niveau du PS II, ceci se traduit par une montée de fluorescence rapide différente lorsqu'on illumine des chloroplastes isolés ou des feuilles (Arntzen et al, 1979);

- dans une feuille sensible ayant absorbé de l'atrazine, la photosynthèse est bloquée et le niveau de fluorescence est maximal. Au contraire, la fluorescence de la feuille résistante est minimale et correspond à celle d'un état normal sans atrazine (Ducruet et Gasquez, 1978);

- on peut définir, pour une solution de chloroplastes une dose qui inhibe $50 \%$ de l'activité $\left(D_{50}\right)$ (Ducruet et Gasquez, 1978). Les chloroplastes sensibles ont une $\mathrm{Dl}_{50}$ d'environ $1^{-7}$ $\mathrm{mol} / \mathrm{l}$ d'atrazine alors que celle des résistants est de $10^{-4} \mathrm{~mol} / \mathrm{l}$;

- enfin, on peut confirmer la présence de la mutation en utilisant l'enzyme de restriction Mae 1 pour digérer un extrait d'ADN chloroplastique : un site de restriction est perdu chez le gène psbA muté (Mc Nally et al, 1987). 


\section{Effets secondaires}

Si l'on considère que la protéine $32 \mathrm{kDa}$ est le site unique de fixation des triazines symétriques, cette mutation apparaît bien être la cause de la résistance des plantes, due à une très grande réduction de l'affinité de la protéine pour l'herbicide. Cependant, d'autres différences apparaissent dans les chloroplastes résistants. En particulier, ceux-ci présentent de plus grands empilements de grana et souvent un rapport chlorophylle a/chlorophylle b réduit (Vaughn et Duke, 1984), ce qui suggèrerait que les antennes du PS II sont plus développées. De plus, un certain nombre de différences lipidiques ont été régulièrement observées en particulier, une augmentation de l'acide C16:1 trans du phosphatidyldiacyl glycérol des résistants (Trémolières et al, 1988). Ces effets pourraient être le résultat de mécanismes de compensation réalisés par la cellule ou l'organisme entier afin d'optimiser une photosynthèse déficiente. En effet, il semble que la modification de la protéine $32 \mathrm{kDa}$ ralentisse le transfert des électrons du PS II. Une réduction du taux d'assimilation du $\mathrm{CO}_{2}$ peut en découler (Holt et al, 1981; Ireland et al, 1988; Ort et al, 1983; Ricroch et al, 1987). D'autres effets secondaires ont été notés sur l'activité des chloroplastes à différentes températures (Darmency et Gasquez, 1981) et sur la stabilité des membranes en réponse à la chaleur (Ducruet et Lemoine, 1985), la concentration en acide abscissique (Touraud et al, 1987) et la distribution journalière des pics de photosynthèse (Dekker et Westfall, 1987).

\section{Valeur adaptative relative des types résistants et sensibles}

En 1979, Conard et Radosevich comparant la croissance de biotypes résistants et sensibles de Senecio vulgaris et Amaranthus retroflexus ont observé que les sensibles se développaient plus rapidement que les résistants et produisaient plus de semences. Ils en concluèrent que la résistance pénalisait les plantes mutées. Une abondante littérature concerne maintenant ce sujet (Holt, 1990).

La majorité des résultats va toujours dans le même sens, mais il existe quelques exceptions où les résistants se développent mieux (chénopodes : Marriage et Warwick, 1980; Jansen et al, 1986; Phalaris paradoxa: Schonfeld et al, 1987).
Cependant nous avons très tôt montré l'intérêt de comparer des plantes isogéniques (backcross, hybrides réciproques, système frère/sœur ou mutant/normal) afin d'éliminer toutes les différences de développement dues à d'autres différences liées au polymorphisme génétique au sein des populations. Ces travaux ne confirment pas systématiquement, pour tous les caractères, des différences entre cytoplasmes résistant et sensible (Stowe et Holt, 1988), mais sur l'ensemble il se dégage une tendance à l'infériorité des résistants (système parent/mutant du chénopode, non publié).

Une partie de cette infériorité peut être liée à la sensibilité à la chaleur des résistants. En effet, chez Polygonum lapathifolium, aucune différence de développement n'a été observée aux basses températures, mais seulement aux températures élevées (Darmency et Gasquez, 1982; Gasquez et al, 1981b). Ceci pourrait avoir des conséquences sur la fréquence des foyers d'infestation selon le climat, mais ce phénomène n'est peutêtre pas généralisable à toutes les espèces (Jacobs et al, 1988).

\section{Origine du phénomène}

Les triazines ne sont pas considérées comme des agents mutagènes (Summer et al, 1984). Aussi, une première hypothèse avancée admet l'existence préalable de mutants à des fréquences très faibles dans les populations, de $10^{-8}$ à $10^{-10}$ (Gressel et Segel, 1978). Ceci pourrait rendre compte de l'apparition de la résistance chez quelques espèces pour lesquelles seulement quelques foyers de résistance ont été observés. Pour les espèces comme Chenopodium album et Senecio vulgaris, le nombre de foyers apparus indépendamment est très grand, ce qui laisse penser à l'existence d'un autre mécanisme. C'est ce qui a été montré en découvrant chez Chenopodium album des plantes particulières donnant un taux élevé de mutants dans leur descendance, $3 \%$ en moyenne (Gasquez et al, 1985).

Ces mutants montrent $100 \%$ de copies d'ADN chloroplastique muté, tandis que leurs plantes sœurs ont $100 \%$ de copies normales. L'activité de leurs chloroplastes en réponse à l'atrazine est identique à celle de chloroplastes des plantes résistantes trouvées dans les champs. Cependant, leur courbe de fluorescence et leur sensibilité au traitement au stade plantule sont intermédiaires 
entre plantes sensibles et résistantes. Par autofécondation, ces mutants donnent une descendance identique à eux-mêmes, mais lorsqu'ils sont soumis à un traitement chimique (un quelconque herbicide à dose sublétale, un fongicide systémique par exemple), leur descendance devient complètement résistante, et ce changement est hérité d'une manière définitive (Bettini et al, 1987). Un second phénomène, inconnu jusqu'à présent, se surajoute donc à la présence du gène chloroplastique $p s b A$ muté.

Ces phénomènes sont encore en cours d'étude. Cependant chez le chénopode, il semble acquis qu'il existe une filiation directe entre les plantes sensibles qui donnent les mutants intermédiaires et les plantes résistantes observés dans les champs. En effet, l'étude génétique des isoenzymes foliaires montre qu'il n'y a qu'un seul génotype résistant par région et que les plantes sensibles qui peuvent donner un fort taux de mutants ont précisément le même génotype dans la même région (Gasquez et Compoint, 1981). Ainsi, en Bourgogne les premières plantes résistantes apparues en 1989 dans du maïs, présentent le même phénotype enzymatique que des plantes sensibles trouvées dès 1980 dans les jardins de Bourgogne et qui ont donné des mutants spontanés au laboratoire.

Dans un champ où existe un génotype particulier, il peut donc apparaître une forte fréquence de mutants, qui, après induction sous l'effet encore inexpliqué de différents produits pesticides, produisent des plantes résistantes. Mais parallèlement, si ce génotype n'existe pas dans ce champ, la résistance a peu de chances d'y apparaître.

\section{LES AUTRES HERBICIDES}

\section{Les bipyridiliums (paraquat)}

Les bipyridiliums sont des herbicides non sélectifs utilisés en traitement du feuillage. Le paraquat est commercialisé depuis le début des années 60 . Le premier cas de résistance au paraquat a été observé chez Poa annua en 1978 dans une pépinière en Grande-Bretagne après 10 ans d'application répétée 2 ou 3 fois par an (Le Baron et Gressel, 1982). Depuis, d'autres cas ont été observés (tableau II), à chaque fois à la suite de nombreux traitements qui ont eu pour conséquence une pression de sélection intense et continue.
Ainsi, la résistance est apparue chez Conyza bonariensis dans des vignes et des plantations d'agrumes en Egypte vers 1979, après environ 9 années de désherbage au paraquat avec 8 passages par an (Harvey et Harper, 1982). Au Japon, des Erigeron résistants sont d'abord apparus vers 1980 dans des plantations de muriers désherbées 2 ou 3 fois par an depuis 1969. Trois espèces d'Erigeron sont maintenant concernées, ainsi que Youngia japonica, toujours dans des cultures pérennes et aussi sur les digues des rizières (Matsunaka et Itoh, 1990). En Australie, 3 espèces ont montré des populations résistantes dès 1983 dans des champs de luzerne traités en hiver, sans rotation de culture ni de labours depuis parfois 20 ans (Powles et Howat, 1990). Enfin, des Conyza ca-

Tableau II. Liste des espèces montrant des populations résistantes à différents herbicides, l'année de la découverte et les pays où des infestations existent. Abréviations : voir légende du tableau 1.

\begin{tabular}{|c|c|c|c|}
\hline Herbicides & Espèces & Année & Pays \\
\hline $\begin{array}{l}\text { Atrazine } \\
\text { (autre que } \\
\text { chloroplastique) }\end{array}$ & $\begin{array}{l}\text { Amaranthus lividus } \\
\text { Abutilon theophrasti } \\
\text { Lolium rigidum } \\
\text { Atriplex patula }\end{array}$ & $\begin{array}{l}1986 \\
1984 \\
1988 \\
1986\end{array}$ & $\begin{array}{l}\text { CH } \\
\text { USA } \\
\text { AU } \\
\text { D, PL }\end{array}$ \\
\hline Paraquat & $\begin{array}{l}\text { Arctotheca calendula } \\
\text { Conyza bonariensis } \\
\text { Conyza canadensis } \\
\text { Erigeron philadelphicus } \\
\text { Erigeron sumatrensis } \\
\text { Hordeum glaucuum } \\
\text { Hordeum leporinum } \\
\text { Poa annua } \\
\text { Youngia japonica }\end{array}$ & $\begin{array}{l}1985 \\
1979 \\
1980 \\
1986 \\
1986 \\
1983 \\
1988 \\
1978 \\
1986\end{array}$ & $\begin{array}{l}\mathrm{AU} \\
\mathrm{ET} \\
\mathrm{J}, \mathrm{H} \\
\mathrm{J} \\
\mathrm{J} \\
\mathrm{AU} \\
\mathrm{AU} \\
\mathrm{GB} \\
\mathrm{J}\end{array}$ \\
\hline Trifluraline & $\begin{array}{l}\text { Eleusine indica } \\
\text { Setaria viridis }\end{array}$ & $\begin{array}{l}1982 \\
1988\end{array}$ & $\begin{array}{l}\text { USA } \\
\text { CND }\end{array}$ \\
\hline Diclofopméthyl & $\begin{array}{l}\text { Avena fatua } \\
\text { Lolium multiflorum } \\
\text { Lolium rigidum }\end{array}$ & $\begin{array}{l}1985 \\
1987 \\
1981\end{array}$ & $\begin{array}{l}\mathrm{AU} \\
\text { USA } \\
\mathrm{AU}\end{array}$ \\
\hline $\begin{array}{l}\text { Chlortoluron } \\
\text { Linuron }\end{array}$ & $\begin{array}{l}\text { Alopecurus myosuroïdes } \\
\text { Conyza canadensis }\end{array}$ & $\begin{array}{r}s 1982 \\
1987\end{array}$ & $\begin{array}{l}\text { GB, D } \\
\mathrm{CH}\end{array}$ \\
\hline Chlorsulfuron & $\begin{array}{l}\text { Kochia scoparia } \\
\text { Lactuca serriola } \\
\text { Salsola iberica } \\
\text { Stellaria media }\end{array}$ & $\begin{array}{l}1987 \\
1986 \\
1988 \\
1988\end{array}$ & $\begin{array}{l}\text { USA } \\
\text { USA } \\
\text { USA } \\
\text { CND }\end{array}$ \\
\hline Mecoprop & $\begin{array}{l}\text { Carduus nutans } \\
\text { Ranunculus acris } \\
\text { Stellaria media }\end{array}$ & $\begin{array}{l}1970 \\
1970 \\
1985\end{array}$ & $\begin{array}{l}\text { NZ } \\
\text { NZ } \\
\text { GB }\end{array}$ \\
\hline $\begin{array}{l}\text { Dérivés } \\
\text { arsénicaux }\end{array}$ & Xanthium strumarium & 1984 & USA \\
\hline Aminotriazole & Lolium rigidum & 1987 & $\mathrm{AU}$ \\
\hline
\end{tabular}


nadensis résistant à la fois au paraquat et à l'atrazine ont été trouvés en 1986 en Hongrie dans des vignobles traités 3 à 4 fois par an au paraquat depuis 10 ans (Pölos et al, 1988).

Le paraquat est un découplant du photosystème $I$ en dérivant le flux d'électrons. Dans les chloroplastes, les radicaux libres de paraquat sont rapidement réoxydés, donnant des superoxydes qui peuvent être des oxydants puissants en formant des radicaux hydroxyle qui causent la péroxydation des acides gras et l'attaque des structures membranaires (Fedkte, 1982). Les mécanismes de résistance ne sont pas encore très clairement démontrés et ils peuvent être différents d'une espèce à l'autre.

Chez Poa annua, la résistance est de l'ordre de 4 fois (plus de $0,8 \mathrm{~kg} / \mathrm{ha}$ ) et s'exprime par des dommages localisés et des repousses non affectées. Pour Conyza bonariensis, la résistance est 5 fois celle des types normaux (insensibilité jusqu'à $10 \mathrm{~kg} / \mathrm{ha}$ ). Deux mécanismes sont proposés pour ce même matériel. D'une part, on enregistre une augmentation de 2 à 3 fois de la quantité des enzymes de détoxication des péroxydes dans les chloroplastes : superoxyde dismutase, ascorbate peroxydase et glutathion réductase (Shaaltiel et Gressel, 1986). C'est aussi ce qui apparaît chez Erigeron philadelphicus où les plantes résistantes restent insensibles à 2 fois la dose qui détruit complètement les plantes sensibles (pas de dégâts jusqu'à $2 \mathrm{~kg} / \mathrm{ha}$ ) et peuvent repousser après traitement à $16 \mathrm{~kg} / \mathrm{ha}$. D'autre part, la résistance peut être due à l'exclusion du produit du site d'action dans le chloroplaste du fait d'une séquestration ou d'une compartimentalisation rapide associée à une mobilité réduite du paraquat : ce pourrait être le mécanisme primaire de la résistance (Fuerst et al, 1985; Fuerst et Vaughn, 1990). De même, un phénomène de séquestration du paraquat dans l'apoplaste des feuilles est invoqué chez Hordeum glaucum (Bishop et al, 1987). Chez cette espèce, la dose létale normale est de $0,05 \mathrm{~kg} / \mathrm{ha}$ tandis que la survie des plantes résistantes n'est pas encore affectée par une dose 32 fois supérieure (Powles, 1986). Enfin, la stimulation des métabolismes de réparation des dégâts implique plusieurs mécanismes, et pourrait être déclenchée par la présence de paraquat comme chez Conyza canadensis qui résiste à $16 \mathrm{~kg} / \mathrm{ha}$ (Pölos et al, 1988).

Quel que soit le mécanisme avancé, 3 études sur Conyza et Hordeum ont montré une hérédité simple de la résistance, avec un seul gène pour lequel l'allèle résistant serait dominant ou codominant (Matsunaka et Itoh, 1990; Islam et Powles, 1988). Par ailleurs, chez Conyza, l'existence de fortes activités des enzymes détoxifiant les radicaux oxygènes est très fortement corrélée à la résistance dans les hybrides entre résistant et sensible et leur descendance (Shaaltiel et al, 1988).

Les résultats d'expériences de compétition inter et intraspécifique chez Hordeum et Erigeron canadensis semblent montrer une plus faible vigueur et une moindre productivité des types résistants en l'absence de paraquat (Tucker et Powles, communication personnelle; Matsunaka et Itoh, 1990).

\section{Dinitroanilines (Trifluraline)}

Des populations d'Eleusine indica insensibles à la trifluraline ont commencé à poser des problèmes dès 1973 en Caroline du Sud (Mudge et al, 1984). Les premières observations ont été faites dans les plantations de coton désherbées pendant 10 ans à la trifluraline. Ce phénomène s'est peu diffusé et concerne seulement 4 états des USA. On peut aussi distinguer un type ayant une résistance intermédiaire. Plus récemment, des populations de Setaria viridis résistantes sont apparues au Manitoba (Canada). Les champs infestés ont subi près de 15 années de désherbage à la trifluraline incorporée en présemis (Morrisson et al, 1989).

Les dinitroanilines agissent sur la division cellulaire, en inhibant la formation des microtubules (Fedtke, 1982). L'étude d'Eleusine indica a montré que le type résistant n'était pas affecté par 10 fois la dose recommandée (pas de dégâts jusqu'à $3 \mathrm{~kg} / \mathrm{ha}$ ) qui tue totalement les plantes sensibles. Dans ce cas, toutes les dinitroaniliniles testées n'ont plus montré d'efficacité, mais il a été observé une activité accrue d'autres molécules affectant les microtubules (Vaughn et al, 1987). Chez le type résistant, il existe une $\beta$ tubuline qui est absente des types sensibles et intermédiaires. Cette nouvelle protéine semble responsable d'une hyperstabilité des microtubules, origine de la résistance : les tubulines des plantes résistantes sont capables de polymériser in vitro en présence du produit toxique (Vaughn et al, 1990).

La résistance chez la sétaire est de 4 à 6 fois plus forte que chez les plantes normales, mais le mécanisme n'est pas encore connu. 
Selon les études en cours, il semble qu'une famille de 4 gènes codant pour les tubulines ait été dupliquée et mutée chez les plantes résistantes (Vaughn, communication personnelle). Ceci aurait entraîné le codage d'une $\beta$-tubuline mutée.

En chambre climatisée, le développement des plantes résistantes est inférieur et montre un rapport de biomasse $R / S$ de 0,69 (Mudge et al, 1984).

\section{Dérivés phénoxy-propioniques (Diclofop-méthyl)}

Depuis 1978, le diclofop-méthyl est largement utilisé en désherbage de post-émergence dans les céréales et les protéagineux. Dès 1981, en Australie, une population de Lolium rigidum était devenue résistante, après seulement 4 années de traitement. Ce phénomène s'est maintenant étendu à de nombreuses populations en Australie. Des populations d'Avena fatua résistantes y sont aussi apparues dès 1985 (Powles et Howat, 1990). Enfin, quelques populations de Lolium multiflorum ont montré une résistance accrue au diclofop en 1987 en Oregon (USA), après 7 ans de traitement sur blé d'hiver (Stanger et Appleby, 1989).

Le diclofop-méthyl touche les étapes enzymatiques de la biosynthèse des acides gras en inhibant l'action de l'acétyl coenzyme A carboxylase des graminées (Secor et al, 1989). Chez les plantes résistantes, il n'a pas été observé d'absorption ni de translocation différentielles. La concentration en acétyl coenzyme A carboxylase n'est pas non plus différente. Cependant, 2 fois plus de diclofop-méthyl semble nécessaire pour inhiber de $50 \%$ l'activité de l'acétyl coenzyme A carboxylase du type résistant chez Lolium rigi$d u m$, mais cela semble trop faible pour justifier de la résistance observée (Powles et al, 1990). En revanche, chez Lolium multiflorum, l'acétyl coenzyme A est environ 15 fois moins sensible chez le type résistant, ce qui peut justifier de la résistance observée (réduction de croissance de $50 \%$ à la dose de $3 \mathrm{~kg} / \mathrm{ha}$, les plantes sensibles étant totalement détruites à des doses de 0,5 à 1 $\mathrm{kg} / \mathrm{ha}$ ) (Stanger et Appleby, 1989; Gronwald et al, 1989).

La conjugaison du diclofop-méthyl avec la glutathion-S-transférase a aussi été examinée chez Lolium rigidum, mais aucune différence n'a été observée entre plantes résistantes et sensibles. En revanche, la détoxication par les mono- oxygénases reste une voie possible pour ce matériel : l'addition d'un inhibiteur de monooxygénases, l'aminobenzotriazole, a un effet synergique du diclofop-méthyl chez les plantes résistantes uniquement, ce qui signifie que la résistance de ces plantes pourrait être partiellement due à une détoxication (Powles et al, 1990). La résistance chez Lolium rigidum se traduit par une insensibilité des populations à $0,4 \mathrm{~kg} / \mathrm{ha}$, dose à laquelle les plantes sensibles sont détruites. Plusieurs populations ne sont pas touchées par des doses jusqu'à $3 \mathrm{~kg} / \mathrm{ha}$. Chez cette espèce, à la différence d'Avena fatua et de Lolium multiflorum, la résistance s'étend à tous les autres dérivés aryloxy-propioniques et on observe des résistances croisées avec d'autres familles d'herbicides ayant des modes d'action différents : sulfonyl-urées, dinitroaniline, cyclohexanedione (Heap et Knight, 1986). Par exemple, on peut trouver parmi les populations résistantes au diclofop-méthyl et n'ayant jamais été soumises à un traitement par une sulfonylurée, une résistance à plus de $0,120 \mathrm{~kg} / \mathrm{ha}$ de chlorsulfuron tandis que $0,020 \mathrm{~kg} / \mathrm{ha}$ détruit complètement les populations sensibles (Heap, 1990). La distribution des résistances croisées est différente d'une population à l'autre. On ne connaît encore rien de l'hérédité de ces phénomènes.

\section{Urées substituées}

Le premier exemple observé provient d'une expérience spécialement conduite pour tester le comportement de Poa annua sous l'effet de 3 ou 4 traitements par an de métoxuron. Après 20 générations, la population, non isolée du pollen des populations voisines, montrait $82 \%$ de plantes survivant au traitement qui en tuait $88 \%$ initialement (Grignac, 1974). La première espèce ayant posé des problèmes en situations agricoles dès 1982 en Angleterre (Moss, 1990) et en Allemagne (Niemann et Pestemer, 1984) est Alopecurus myosuroides. Plusieurs autres populations sont maintenant concernées dans 6 comtés en Angleterre. Elles proviennent de champs conduits surtout en succession de céréales d'hiver, sans labour, avec en moyenne 1,6 traitement par an au chlortoluron ou à l'isoproturon depuis 10 ans. Enfin, citons Conyza canadensis résistant au linuron dans différentes cultures de carottes en Suisse et rappelons l'existence de résistances croisées variables avec la résistance chloroplastique à l'atrazine (Beuret, 1988). 
Les urées substituées qui persistent longtemps dans le sol sont absorbées par les racines et agissent essentiellement en inhibant la photosynthèse (Fedtke, 1982).

Chez Poa annua, on sait seulement que la moitié des plantes qui résistaient à des doses normales d'utilisation étaient détruites à fortes doses $(6 \mathrm{~kg} / \mathrm{ha})$. Chez Conyza canadensis, la résistance, d'environ 4 à 5 fois sur plante entière, n'est pas liée à une modification du chloroplaste, mais n'a pas été plus étudiée (Beuret, 1988). Des études plus complètes ont permis de mieux décrire la résistance d'Alopecurus myosuroides. Cette résistance varie considérablement entre populations et même d'une plante à l'autre. La mortalité chez les résistants commence avec 3 $\mathrm{kg} / \mathrm{ha}$ de chlorotoluron et des plantes survivent encore après $7 \mathrm{~kg} / \mathrm{ha}$, tandis que les plantes sensibles sont complètement détruites avec 1 $\mathrm{kg} / \mathrm{ha}$. Cette différence ne tient pas à une absorption ou translocation différente mais à une dégradation supérieure de l'herbicide chez les plantes résistantes. La détoxication fait intervenir les mono-oxygénases liées au cytochrome $P$ 450 (Kemp et al, 1990). Cette résistance s'étend à plusieurs urées siabstituées (isoproturon) et à d'autres herbicides comme des phénoxypropioniques (diclofop), des dinitroanilines (pendimethaline) et imidazolinones (imazamethabenz), mais l'intensité de ces résistances croisées peut varier d'une population à une autre et peut être faible, ce qui laisse penser que différents mécanismes de détoxication peuvent exister.

Des expériences préliminaires chez Alopecurus myosuroides montrent que la résistance peut être transmise via le pollen et que différents niveaux de résistance s'expriment dans la descendance de ces plantes. Ceci indique une hérédité nucléaire polygénique. Du fait d'une allogamie importante, une population résistante au champ peut échanger des gènes avec les populations sensibles voisines et donc, produire une descendance composée de différents types de semences, de sensibles à plus ou moins résistantes. Aucune différence de compétitivité ni de croissance n'a été détectée entre les plantes résistantes et sensibles (Moss, 1990).

\section{Sulfonyl-urées}

Le chlorsulfuron a été commercialisé aux USA et en Australie dès 1982 pour le désherbage des céréales. II agit en inhibant la synthèse de certains acides aminés via l'inhibition de l'acétolac- tate synthase (Chaleff et Mauvais, 1984). Les premiers problèmes sont apparus en 1986 en Idaho avec Lactuca serriola, puis se sont rapidement multipliés avec 2 autres espèces dans l'Ouest et le Sud des USA dans les zones de cultures extensives : Kochia scoparia et Salsola iberica. De plus, des Stellaria media ont été reconnues résistantes au Canada (Mallory-Smith et al, 1990). Enfin, mentionnons la résistance croisée chez Lolium rigidum en Australie.

Les plantes de Lactuca serriola résistent à $53 \mathrm{~g} / \mathrm{ha}$ d'un mélange metsulfuron/chlorsulfuron (1/2), tandis que la dose d'utilisation ne dépasse pas $20 \mathrm{~g} / \mathrm{ha}$. La résistance n'est pas due à une absorption, une translocation ou un métabolisme différent. II s'agit d'une modification de la cible, l'enzyme acétolactate synthase, qui est moins inhibé chez les plantes résistantes (rapport d'inhibition $50 \%: R / S=17$ ) (Thill et al, non publié). II existe une résistance croisée pour les autres sulfonylurées et les imidazolinones.

Cette résistance semble héritée via un simple gène nucléaire dominant ou non selon les conditions du milieu (on observe dans la génération $F_{2}$ d'un croisement résistant $x$ sensible, soit $3 / 4$, soit $1 / 4$ de type résistant selon la localité d'expérimentation) (Thill et al, non publié).

\section{Phytohormones}

À la suite d'une baisse d'efficacité du mécoprop sur des Stellaria media dans 2 champs en Angleterre, il a été montré qu'elles étaient devenues résistantes à ce produit. Ce phénomène n'était perceptible que dans les céréales lorsqu'aucun autre herbicide n'était utilisé (Lutman et Lovegrove, 1985). Aux doses normales d'utilisation, les plantes résistantes sont peu affectées. À des doses plus fortes, elles montrent des symptômes sur les feuilles et les pétioles mais ces dégâts disparaissent par la suite. La résistance est d'environ 6 fois supérieure à celle des plantes normales. Le mécanisme pourrait être lié à des affinités différentes du mécoprop sur les récepteurs hormonaux chez ces plantes (Barnwell et Cobb, 1989). II semble pour cette espèce que la résistance puisse exister dans certaines lignées indépendamment du traitement. L'apparition de résistances aux phytohormones semble très lente. Deux autres cas sont cités dans les prairies de Nouvelle-Zélande, Ranunculus acris et Carduus nutans. Des populations de ces espèces ont commencé à poser irrégulièrement quelques problèmes dans les années 1970. Après expérimen- 
tation au champ ou en serre, les populations résistantes issues de milieux traités depuis longtemps supportaient en moyenne 5 à 6 fois plus de MCPA que des populations témoins. Des traitements à $1 \mathrm{~kg} / \mathrm{ha}$ de MCPA tuaient $100 \%$ des témoins mais très peu d'individus des populations sélectionnées (Bourdot et al, 1989).

\section{Dérivés arsenicaux}

Les sels de l'acide méthylarsenic (DSMA et MSMA) sont utilisés aux USA pour le désherbage en post-émergence de Xanthium strumarium dans les plantations de coton. Depuis 15 ans, des champs ont reçu 1 à 2 traitements de ces produits par an. En 1984, les traitements sur plusieurs populations de Xanthium n'ont causé aucun effet phytotoxique. Des dommages apparaissent pour $2,2 \mathrm{~kg} / \mathrm{ha}$ tandis que toutes les plantes sensibles sont tuées avec $1,1 \mathrm{~kg} / \mathrm{ha}$ (Hajgler et al, 1988).

\section{Aminotriazole}

L'aminotriazole est un produit de contact qui agit via une interaction avec la synthèse des caroténoïdes (Fedtke, 1982). Le cas de sélection d'un Lolium rigidum sur les voies ferrées en Australie par un mélange aminotriazole - triazine a été signalé dès 1987, soit après 10 années de traitement (1 par an). L'étude du mécanisme mis en cause est en cours (Powles et Howat, 1990). II y a résistance croisée avec toutes les triazines symétriques et les urées substituées.

\section{STRATÉGIES POUR COMBATTRE CES RÉSISTANCES}

Toutes les résistances décrites jusqu'à ce jour, à l'exception peut-être de Stellaria media résistante aux phytohormones, sont survenues à la suite d'un nombre important de traitements qui ont créé une pression de sélection puissante et quasi permanente. Même dans le cas d'herbicides de contact sans persistance, comme le paraquat, on observe le même résultat à la suite de plusieurs passages annuels, jusqu'à 8 ! On peut donc penser qu'un système de rotation des familles d'herbicides peut empêcher ou retarder l'apparition des conditions ad hoc pour la sélection de la résistance. C'est ce que prédisent les premiers mo- dèles de dynamique des populations résistantes (Gressel, 1990), mais aucun résultat d'expérimentation n'a encore été publié à ce sujet.

Cependant, il faut remarquer qu'un grand nombre de champs ayant subi les mêmes désherbages intensifs sont encore indemnes de plantes résistantes. L'exemple de Chenopodium album résistant à l'atrazine montre que la présence initiale de plantes sensibles ayant un taux élevé de mutants, est d'une grande importance pour l'évolution de la population. La distribution des mutants n'est pas forcément uniforme sur le territoire ni même aléatoire, elle peut être agrégée et donc laisser des zones indemnes. II faut donc éviter toute contamination des champs indemnes par des semences ou du pollen issus de champs infestés, car l'évolution vers la résistance n'est pas inéluctable. Machines agricoles et épandages de lisier ou de compost sont les principales sources de contamination.

L'aspect génétique de la réponse des mauvaises herbes à leur environnement intervient aussi dans l'existence plutôt généralisée d'une valeur adaptative moindre des types résistants dont la survie est plus faible en l'absence de l'herbicide concerné. Ceci souligne l'intérêt des rotations de cultures et d'herbicides afin de ménager, en théorie, des phases de décroissance du nombre de plantes résistantes. En effet, quelques plantes résistantes ne font pas une population résistante et ne causent pas de problèmes à l'agriculteur. II importe donc de savoir surveiller les populations de mauvaises herbes et de les gérer via les pratiques culturales et l'utilisation de modèles d'évolution. Ces modèles doivent intégrer nos connaissances sur le développement des plantes, leur interaction avec la culture, leur reproduction et la survie des semences dans le sol.

Devant une infestation par une population résistante, la réponse peut être de changer d'herbicide. Ce n'est pas toujours facile, surtout si la même culture est conservée. Ainsi, la résistance aux triazines a été combattue chez le maïs uniquement en réalisant un désherbage de postémergence supplémentaire, ce qui entraîne un surcoût important sans réduire notablement les populations résistantes au fil des années puisque l'on continue à utiliser l'herbicide responsable de la sélection. Le problème des résistances croisées entre produits ayant des modes d'action différents est très préoccupant, car il indique qu'il peut exister un mécanisme général de détoxication et donc fortement limiter la gamme des herbicides de remplacement. $\mathrm{Ce}$ - 
pendant, on peut essayer d'exploiter le phénomène inverse, à savoir les résistances négatives. Par exemple, les plantes résistantes à l'atrazine sont toujours plus sensibles aux colorants nitrés. Des synergies entre produits sont aussi connues, ce qui permettrait de réduire les doses de ces herbicides et de diminuer leur pression de sélection (Hatzios et Penner, 1985). Enfin, la rotation d'herbicides doit prendre en compte non seulement le mode d'action différent, mais aussi un type de dégradation et de détoxication différents, et réexaminer les sélectivités dites de position.

Cependant, l'apparition d'infestations de plantes résistantes aux herbicides n'est pas limitée aux systèmes intensifs de culture. L'extensification, avec ou sans jachère, ne pourra se passer à moyen terme de l'utilisation intégrée d'herbicides. Le faible coût obligatoire des intrants réduira le choix de l'agriculteur aux mêmes herbicides dont il répètera l'utilisation. C'est dans de telles situations qu'on a déjà observé des apparitions rapides de résistances : sulfonylurées aux États-Unis et résistances croisées en Australie.

Enfin, remarquons que l'optimisation du désherbage chimique peut être réalisée grâce à l'emploi de cultivars résistants, évitant la phytotoxicité préjudiciable tout en maintenant au niveau désiré la densité des mauvaises herbes. La création par amélioration des plantes classique ou par génie génétique, est un domaine très actif actuellement (Darmency, 1984; Mazur et Falco, 1989). II devrait permettre l'utilisation des herbicides non sélectifs sans risque pour la culture. Cependant, on peut craindre que des échanges géniques par introgression se réalisent entre plantes cultivées et plantes sauvages affines, le résultat étant la diffusion de résistances nouvelles parmi les mauvaises herbes et, par voie de conséquence, la réduction de l'intérêt d'un tel système de culture.

Pour conclure, soulignons que si l'apparition de résistances aux herbicides est moins préoccupante en France que pour les fongicides ou les acaricides, il ne faut pas pour autant la négliger ou la considérer comme inéluctable. Au contraire, elle doit, dès à présent, nous obliger à mettre au point des solutions alternatives de désherbage alors que les contraintes du système agricole se font plus restrictives (marges financières, protection de l'environnement). Nous sommes encore loin de 600 espèces d'insectes résistant aux insecticides, mais nous pouvons déjà profiter des enseignements tirés de la lutte contre ces insectes.
II se dégage de cette étude qu'un effort de prévention important doit être mis en œuvre dès à présent. Plusieurs équipes de chercheurs se penchent actuellement sur ce sujet, essentiellement en essayant de mieux comprendre les différentes phases de la biologie des mauvaises herbes et en adaptant des modèles mathématiques prévisionnels (Roush et al, 1990; Putwain et al, 1990; Gressel, 1990). Si l'apparition de mutants ne peut être évitée, on peut essayer de mieux gérer les pratiques culturales (par des rotations de cultures, d'herbicides, voire l'introduction du sarclage) afin de limiter le développement de ces mutants et d'empêcher la formation de populations résistantes posant de véritables problèmes.

\section{RÉFÉRENCES}

Andersen RN, Gronwald JW (1987) Noncytoplasmic inheritance of atrazine tolerance in velvetleaf (AbutiIon theophrasti). Weed Sci 35, 496-498

Arntzen CJ, Ditto CL, Brewer PE (1979) Chloroplast membrane alteration in triazine-resistant Amaranthus retroflexus biotypes. Proc Natl Acad Sci USA $76,278-282$

Barnwell P, Cobb AH (1989) Physiological studies of mecoprop-resistance in chickweek (Stellaria media L). Weed Res 29, 135-140

Bettini P, McNally S, Sevignac M, Darmency H, Gasquez J, Dron M (1987) Atrazine resistance in Chenopodium album : Low and high levels of resistance to the herbicide are related to the same chloroplast psbA gene mutation. Plant Physiol 84, 1442-1446

Beuret $E$ (1988) Cas particuliers de résistance à l'atrazine et au linuron chez Amaranthus lividus $L$ et Erigeron canadensis L. VIIIe Colloque International Biologie Écologie Système des Mauvaises Herbes, 277-285

Bishop T, Powles SB, Cornic G (1987) Mechanism of paraquat resistance in Hordeum glaucum II. Paraquat uptake and translocation. Aust $J$ Plant Physiol 14, 539-547

Bourdot GW, Harrington KC, Popay Al (1989) The appearance of phenoxy-herbicide resistance in New Zeland pasture weeds. Brighton Crop Protec Conf Weeds, 309-316

Bowes J, Crofts AR, Arntzen CJ (1980) Redox reactions on the reducing side of photosystem II in chloroplasts with altered herbicide binding properties. Arch Biochem Biophys 200, 303-308

Bulke R, de Praeter H, Van Himme M, Stryckers J (1984) Resistance of annual meadow-grass, Poa annua L, to 2-chloro-1,3,5-triazines. Meded Fac Landbouwwet Rijksuniv Gent 49, 1041-1050

Chaleff RS, Mauvais CJ (1984) Acetolactate synthase is the site of action of two sulfonylurea herbicides in higher plants. Science 224, 1443-1445 
Cheung AY, Bogorad L, Montagu MV, Schell J (1988) Relocating a gene for herbicide tolerance : a chloroplast gene is converted into a nuclear gene. Proc Natl Acad Sci USA 85, 391-395

Conard SG, Radosevich SR (1979) Ecological fitness of Senecio vulgaris and Amaranthus retroflexus biotypes susceptible or resistant to atrazine. J Appl Ecol 16, 171-177

Darmency H (1984) Sélection pour la résistance aux herbicides. Le Sélectionneur Français 33, 47-53

Darmency H, Gasquez J (1981) Inheritance of triazine resistance in Poa annua: consequences for population dynamics. New Phytol 89, 487-493

Darmency H, Gasquez J (1982) Differential temperature - dependance of the Hill activity of isolated chloroplasts from triazine resistant and susceptible biotypes of Polygonum lapathifolium L. Plant Sci Lett 24, 39-44

Darmency H, Gasquez J (1983) Interpreting the evolution of a triazine-resistant population of Poa annua L. New Phytol 95, 299-304

Darmency H, Gasquez J (1990) The fate of herbicide resistance genes in weeds. In: Managing Resistance to Agrochemicals. (Green MB, Moberg WK, LeBaron HM, eds) ACS Symp Ser 421, Washington, 353-363

Dekker J, Westfall B (1987) A temporal phase mutation of chlorophyll fluorescence in triazine-resistant Brassica napus. Z Naturforsch Sect C Biosci 42, 775-778

Demeter S, Vass I, Hideg E, Sallai A (1985) Comparative thermoluminescence study of triazine-resistant and -susceptible biotypes of Erigeron canadensis $L$. Biochim Biophys Acta 806, 16-24

Ducruet JM \& Gasquez J (1978) Observations de la fluorescence sur feuille entière et mise en évidence de la résistance chloroplastique à l'atrazine chez Chenopodium album L et Poa annua L. Chemosphere 8, 691-696

Ducruet JM, Lemoine $Y$ (1985) Increased heat sensitivity of the photosynthetic apparatus in triazineresistant biotypes from different plant species. Plant Cell Physiol 26, 419-429

Ducruet JM, de Prado R (1982) Comparison of inhibitory activity of amides derivatives in triazineresistant and -susceptible chloroplasts from Chenopodium album and Brassica campestris. Pestic Biochem Physiol 18, 253-261

Ellis M, Kay QON (1975) Genetic variation in herbicide resistance in scentless mayweed (Tripleurospermum inodorum L Schultz Bip) I. Difference between populations in response to MCPA. Weed Res 15, 285-294

Fedtke C (1982) Biochemistry and Physiology of Herbicide action. Springer-Verlag, Berlin, $202 \mathrm{p}$

Fogelfors $H$ (1979) Changes in the flora of farmland. Swed Univ Agric Sci Dep Ecol Environ Res Rep 5, $66 \mathrm{p}$
Fuerst EP, Vaughn KC (1990) Mechanism of Paraquat resistance (Caseley J, ed) Butterworths. Weed Technol 4 (sous presse)

Fuerst EP, Nakatani HY, Dodge AD, Penner D, Arntzen CJ (1985) Paraquat resistance in Coniza. Plant Physiol 77, 984-989

Fuerst EP, Arntzen CJ, Pfister K, Penner D (1986) Herbicide cross-resistance in triazine-resistant biotypes of four species. Weed Sci 34, 344-353

Gasquez J (1981) Évolution de la résistance aux triazines chez les espèces annuelles : la résistance chloroplastique. Comptes Rendus $11^{\mathrm{e}}$ Conférence Columa, Versailles, 4, 981-1006

Gasquez J, Barralis G, Aigle N (1982) Distribution et extension de la résistance chloroplastique aux triazines chez les adventices annuelles en France. Agronomie 2, 119-124

Gasquez J, Compoint JP (1973) Étude préliminaire de la variation infraspécifique chez Echinochloa crus galli (L) PB Comptes Rendus Vle Colloque International Biologie Écologie Système des Mauvaises Herbes, Marseille, 33-50

Gasquez J, Compoint JP (1981) Enzymatic variations in population of Chenopodium album $\mathrm{L}$ resistant and susceptible to triazine. Agro-Ecosystems 7, 110

Gasquez J, Darmency H (1983) Variation for chloroplast properties between two triazine resistant biotypes of Poa annua L. Plant Sci Lett 30, 99-106

Gasquez J, Darmency H (1990) Herbicide resistance in France. In: Importance and perspectives on herbicide resistant weeds (Cavalloro $\mathrm{R}$, Noyé $\mathrm{G}$, eds) EUR 11561, Agriculture series, Luxembourg, 81-94

Gasquez J, Darmency H, Compoint JP (1981a) Étude de la transmission de la résistance chloroplastique aux triazines chez Solanum nigrum. CR Acad Sci Ser D 292, 847-849

Gasquez J, Darmency H, Compoint JP (1981b) Comparaison de la germination et de la croissance de biotypes résistants et sensibles aux triazines chez quatre espèces de mauvaises herbes. Weed Res $21,219-225$

Gasquez J, Al Mouemar A, Darmency H (1984) Quels gènes pour la résistance chloroplastique aux triazines chez Chenopodium album L? VIle Colloque International Écologie Biologie Système des Mauvaises Herbes, 281-286

Gasquez J, Al Mouemar A, Darmency H (1985) Triazine herbicide resistance in Chenopodium album $L$. Occurence and characteristics of an intermediate biotype. Pestic Sci 16, 390-395

Gennaro (de) FP, Weller SC (1984) Differential susceptibility of field bindweed (Convolvulus arvensis) biotypes to glyphosate. Weed Sci 32, 472-476

Gressel J (1990) Prevention and management of herbicide resistance : theorical aspects. Weed Technol 4, 186-198

Gressel J, Segel LA (1978) The paucity of plants evolving genetic resistance to herbicides : possible reasons and implications. $J$ Theor Biol 75, 349-372 
Grignac P (1974) Sélection d'un biotype de paturin annuel (Poa annua $L$ ) resistant au métoxuron par répétition de traitements herbicides. CR Acad Agric Fr 60, 401-408

Gronwald JW, Eberlein CV, Betts KJ, Rosow KM, Ehlke NJ, Wyse DL (1989) Diclofop resistance in a biotype of italian ryegrass. Plant Physiol 89-115

Haigler WE, Gossett BJ, Harris JR, Toler JE (1988) Resistance of common cocklebur (Xanthium strumarium) to the organic arsenical herbicides. Weed Sci 36, 24-27

Harvey BMR, Harper BD (1982) Tolerance to bipyridylium herbicides. In: Herbicide resistance in Plants (Le Baron H, Gressel J, eds) Wiley, NY 215-233

Hatzios KK, Penner D (1985) Interaction of herbicides with other agrochemicals in higher plants. Rev Weed Sci 1, 1-63

Heap IM (1990) Resistance to herbicide in annual ryegrass (Lolium rigidum). In: Herbicide resistance in weeds and crops (Caseley J, ed) Butterworths (sous presse)

Heap I, Knight R (1986) The occurrence of herbicide cross-resistance in a population of annual ryegrass, Lolium rigidum, resistant to diclofop methyl. Aust $J$ Agric Res 37, 149-156

Hirshberg J, McIntosh L (1983) Molecular basis of herbicide resistance in Amaranthus hybridus. Science $22,1346-1349$

Holt JS (1990) Fitness and ecological adaptability of herbicide resistant biotypes. In: Managing Resistance to Agrochemicals (Green MB, Moberg WK, LeBaron HM, eds) ACS Symp Ser 421, Washington, 419-429

Holt JS, Stemler AJ, Radosevich R (1981) Differential light responses of photosynthesis by triazineresistant and triazine-susceptible Senecio vulgaris biotypes. Plant Physiol 67, 744-748

Ireland CR, Telfer A, Covello PS, Baker NR, Barber J (1988) Studies on the limitations to photosynthesis in leaves of the atrazine-resistant mutant of Senecio vulgaris L. Planta (Berl) 173, 459-467

Islam AKMR, Powles SB (1988) Inheritance of resistance to paraquat in barley grass Hordeum glaucum Steud. Weed Res 28, 393-397

Jacobs BF, Duesing JH, Antonovics J, Patterson DT (1988) Growth performance of triazine-resistant and -susceptible biotypes of Solanum nigrum over a range of temperatures. Can J Bot 66, 847-850

Jana S, Naylor JM (1982) Adaptation for herbicide tolerance in populations of Avena fatua. Can $J$ Bot $60,1611-1617$

Jansen MAK, Hobe JH, Wesselius JC, Van Rensen JJS (1986) Comparison of photosynthetic activity and growth performance in triazine-resistant and susceptible biotypes of Chenopodium album. Physiol Vég 24, 475-484

Jursinic P, Stemler A (1983) Changes in ${ }^{14} \mathrm{C}$ atrazine binding associated with the oxidation-reduction state of the secondary quinone acceptor of photosystem II. Plant Physiol 73, 703-708
Karim A, Bradshaw AD (1968) Genetic variation in simazine resistance in wheat, rape and mustard. Weed Res 8, 283-291

Kemp MS, Moss SR, Thomas TH (1990) Herbicide resistance in Alopecurus myosuroides. In: Managing Resistance to Agrochemicals (Green MB, Moberg WK, LeBaron HM, eds) ACS Symp Ser 421, Washington, 376-393

Le Baron HM, Gressel J (1982) Herbicide resistance in plants. Wiley \& Sons, New York, $401 \mathrm{p}$

Le Baron HM, McFarland J (1990) Overview and prognosis of herbicide resistance in weeds and crops. In: Managing Resistance to Agrochemicals (Green MB, Moberg WK, Le Baron HM, eds) ACS Symp Ser 421, Washington, 336-352

Lutman PJW, Lovegrove AW (1985) Variations in the tolerance of Galium aparine (cleavers) and Stellaria media (chickweed) to mecopcrop. Proc Brit Crop Prot Conf Weeds, Brighton, 411-418

Mallory-Smith CA, Thill DC, Dial MJ (1990) Identification of sulfonylurea herbicide-resistant prickly lettuce (Lactuca serriola). Weed Technol 4, 163-168

Marriage PB, Warwick SI (1980) Differential growth and response to atrazine between and within susceptible and resistant biotypes of Chenopodium album L. Weed Res 20, 9-15

Matsunaka S, Itoh K (1990) Paraquat resistance in Japan. In: Herbicide resistance in weeds and crops (sous presse)

Mazur BJ, Falco SC (1989) The development of herbicide resistant crops. Annu Rev Plant Physiol Plant Mol Biol 40, 441-470

Morrisson IN, Todd BG, Nawolsky KM (1989) Confirmation of trifluralin resistant green foxtail (Setaria viridis) in Manitoba. Weed Technol 3, 544-551

Moss SR (1990) Herbicide resistance in slender foxtail (Alopecurus myosuroides Huds) cross-resistance and population studies. Weed Technol 4 (sous presse)

Mudge LC, Gosset BJ, Murphy TR (1984) Resistance of goosegrass (Eleusine indica) to dinitroaniline herbicides. Weed Sci 32, 591-594

McNally S, Bettini P, Sevignac M, Darmency $H$, Gasquez J, Dron M (1987) A rapid method to test for chloroplast DNA involvement in atrazine resistance. Plant Physiol 83, 248-250

My J, Lorelle V (1988) Utilisation des produits phytosanitaires en France. Phytoma Déf Cult 399, 8-10

Nieman P, Pestemer W (1984) Resistenz verschiedener herkunfte von Acker-Fichsschwanz (Alopecurus myosuroides) gegenuber herbizidbehandlungen. Nachrichtenbl Dtsch Pflanzenschutzdienst (Braunschweig) 36, 113-118

Ort DR, Ahrens WH, Martin BH, Stoller EW (1983) Comparison of photosynthetic performance in triazine-resistant and susceptible biotypes of Amaranthus hybridus. Plant Physiol 72, 925-930

Peabody D (1974) Herbicide tolerant weeds appear in western Washington. Weeds Today 5, 14 
Pfister K, Steinback KE, Gardner G, Arntzen CJ (1981) Photoaffinity labeling of an herbicide receptor protein in chloroplast membranes. Proc Natl Acad Sci USA 78, 981-985

Pölos E, Mikulas J, Szigeti Z, Matkovics B, Do Quy Hai, Parducz A, Lehoczki E (1988) Paraquat and atrazine co-resistance in Conyza canadensis (L) Cronq Pestic Biochem Physiol 30, 142-154

Powles SB (1986) Appearance of a biotype of the weed, Hordeum glaucum Steud resistant to the herbicide paraquat. Weed Res 26, 167-172

Powles SB, Howat PD (1990) Herbicide resistant weeds in Australia. Weed Technol 4, 178-185

Powles SB, Holtum JAM, Matthews JM, Liljegren DR (1990) Multiple herbicide resistance in annual ryegrass (Lolium rigidum) : the search for a mechanism. In: Managing Resistance to Agrochemicals (Green MB, Moberg WK, LeBaron HM, eds) ACS Symp Ser 421, Washington, 394-406

Price SC, Hill JE, Allard RW (1983) Genetic variability for herbicide reaction in plant populations. Weed Sci 31, 652-657

Putwain PD, Mortimer AM, Ulf-Hansen PF, Watson D (1990) Population ecology and selection for herbicide-resistance. Weed Technol 4 (sous presse)

Rao JKM, Hargrave PA, Argos P (1983) Will the seven-helix bundle be a common structure for integral membrane proteins ? Febs Lett 156, 165-169

Ricroch A, Mousseau M, Darmency H, Pernes J (1987) Comparison of triazine-resistant and susceptible cultivated Setaria italica L (PB): growth and photosynthetic capacity. Plant Physiol Biochem $25,29-34$

Roush ML, Radosevick SR, Maxwell BD (1990) Future outlook for herbicide-resistance research. Weed Technol 4, 208-214

Rubin B, Yaacoby T, Schonfeld M (1985) Triazine resistant grass weeds: cross resistance with wheat herbicide, a possible threat to cereal crops. Brighton Crop Prot Conf Weeds 11, 1171-1179

Ryan GF (1970) Resistance of common groundsel to simazine and atrazine. Weed Sci 18, 614-616

Schonfeld M, Yaacoby T, Michael O, Rubin B (1987) Triazine resistance without reduced vigor in Phalaris paradoxa. Plant Physio/ 83, 329-333

Secor J, Cséké C, Owen WJ (1989) The discovery of the selective inhibition of acetyl coenzyme A carboxylase activity by two classes of graminicides. Proc Brit Crop Protec Conf Weed, Brighton,145-154

Shaaltiel Y, Gressel J (1986) Multienzyme oxygen radical detoxifying system correlated with paraquat resistance in Conyza bonariensis. Pestic Biochem Physiol 26, 22-28

Shaaltiel Y, Chua N-H, Gepstein S, Gressel J (1988) Dominant pleiotropy controls enzymes cosegregating with paraquat resistance in Conyza bonariensis. Theor App/ Genet 75, 850-856

Shimabukuro RH, Kadunce KE, Frear DS (1966) Dealkylation of atrazine in mature pea plants. $J$ Agric Food Chem 14, 393-395
Souza-Machado V, Bandeen JD, Stephenson GR, Jensen KIN (1977) Differential atrazine interference with the Hill reaction of isolated chloroplasts from Chenopodium album L biotypes. Weed Res $17,407-413$

Souza-Machado V, Arntzen CJ, Bandeen JD, Stephenson GR (1978a) Comparative triazine effects upon system II photochemistry in chloroplasts of two common lambsquarters (Chenopodium album) biotypes. Weed Sci 26, 318-322

Souza-Machado V, Bandeen JD, Stephenson GR, Lavigne $P$ (1978b) Uniparental inheritance of chloroplast atrazine tolerance in Brassica campestris. Can J Plant Sci 58, 977-981

Stanger CE, Appleby AP (1989) Italian ryegrass (Lolium multiflorum) accessions tolerant to diclofop. Weed Sci 37, 350-352

Stowe AE, Holt JS (1988) Comparison of triazineresistant and -susceptible biotypes of Senecio vulgaris and their $F_{1}$ hybrids. Plant Physiol 87, 183189

Summer DD, Cassidy JE, Szolics IM, Marco GJ, Bakshi KS, Brusick DJ (1984) Evaluation of the mutagenic potential of corn Zea mais $L$ grown in untreated and atrazine treated soils in the field. Drug Chem Toxicol 7, 243-257

Touraud G, Leydecker MT, Darmency H (1987) Abscissic acid in triazine resistant and susceptible Poa annua. Plant Sci 49, 81-83

Trebst A (1987) The three-dimensional structure of the herbicide binding niche on the reaction center polypeptides of photosystem II. Z Naturforsch Sect C Biosci 42, 742-750

Tremolières A, Darmency $H$, Gasquez J, Dron $M$, Connan A (1988) Variation of transhexadecenoic acid content in two triazine resistant mutants of Chenopodium album and their susceptible progenitor. Plant Physiol 86, 967-970

Vaughn KC, Vaughn MA, Gosset BJ (1990) Dinitroaniline resistance mechanism and characteristics of resistance. Weed Techno/ 4, 157-162

Vaughn KC, Duke SO (1984) Ultrastructural alterations to chloroplasts in triazine-resistant weed biotypes. Physiol Plant 62, 510-520

Vaughn KC, Marks MD, Weeks DP (1987) A dinitroaniline-resistant mutant of Eleusine indica exhibits cross-resistance and supersensitivity to antimicrotubule herbicides and drugs. Plant Physiol 83, 956964

Warwick DD (1976) Plant variability in triazine resistance. Residue Rev 65, 56-63

Warwick SI, Marriage PB (1982) Geographical variation in populations of Chenopodium album resistant and susceptible to atrazine. I. Between and within population variation in growth and response to atrazine. Can J Bot 60, 483-493

Zanin G, Vecchio V, Gasquez J (1981) Indagini sperimentali su populazioni di dicotiledoni resistenti all'atrazina. Riv Agron 3-4, 196-207 